\title{
Identification of irradiated dried fruits using EPR spectroscopy
}

Grzegorz P. Guzik, Wactaw Stachowicz, Jacek Michalik

\begin{abstract}
The dominating carbohydrates in fruits are monosaccharides like fructose, glucose, sorbose and mannose. In dehydrated fruits, concentration of monosaccharides is higher than in fresh fruits resulting in the formation of sugar crystallites. In most of dried fruits, crystalline fructose, and glucose dominate and appear in proportion near to 1:1. Irradiation of dried fruits stimulates radiation chemical processes resulting in the formation of new chemical products and free radicals giving rise to multicomponent EPR signal which can be detected for a long period of time. For that reason, it is used as a marker for the detection of radiation treatment of dried fruits. It has been found that EPR spectra recorded in dried banana, pineapple, papaya, and fig samples resemble the EPR spectrum obtained by computer addition of fructose and glucose spectra taken in proportion $1: 1$. The decay of radiation induced EPR signals proceeds in dried fruits fast during the first month of observation and becomes much slower and almost negligible after prolonged storage. However, it remains intense enough for EPR detection even one year after processing. The radiation induced EPR signal is easily detected in dried fruits exposed to $0.5 \mathrm{kGy}$ of gamma rays. Thus, the EPR method of the detection of irradiated fruits can be used for the control of dried fruits undergoing quarantine treatment with 200-300 Gy of ionizing radiation.
\end{abstract}

Key words: dried fruits $\bullet$ electron paramagnetic resonance $(\mathrm{EPR}) \bullet$ fructose $\bullet$ glucose $\bullet$ irradiation

G. P. Guzik ${ }^{\bowtie}$, W. Stachowicz, J. Michalik Institute of Nuclear Chemistry and Technology, 16 Dorodna Str., 03-195 Warsaw, Poland, Tel.: +48 22504 1122, Fax: +48 22811 1532, E-mail: g.guzik@ichtj.waw.pl

Received: 3 November 2014

Accepted: 20 May 2015

\section{Introduction}

First information on stable EPR signals observed in biological tissues and food exposed to ionizing radiation appeared in the sixties of the last century [1]. Dodd et al. reported specific EPR signal in seeds of irradiated strawberries [2]. Raffi and coworkers observed stable, multiline EPR signals in dried fruits like figs, bilberries, raspberries and red currants exposed to ionizing radiation [3]. The author suggested that multicomponent EPR signal observed in dried fruits exposed to radiation is assigned to radicals trapped in crystalline sugars [4-6]. Stability and specificity of these signals make them suitable for control whether dried fruits were irradiated or not [7]. The reliability of these EPR signals as indicators of irradiation of dried fruits has been proven in a number of interlaboratory tests [5, 8]. Consequently, the European standard EN-13708 "Foodstuffs - Detection of irradiated food containing crystalline sugar by ESR spectroscopy" has been issued by CEN (Committee European de Normalisation in Brussels) in 2002 [9]. The standard states: "Irradiated food containing crystalline sugar show typical multicomponent ESR spectra reflecting the 
presence of radiation-induced radicals in the sample. Dried fruits often contain sugar particles in crystalline form, and therefore the appearance of a typical multicomponent ESR spectrum indicates radiation treatment. Due to different saccharide composition the shape of recorded EPR spectra differs for various dried fruits". The radiation dose recommended by WHO/FAO to eliminate pests and insects from dried fruits is in the range $0.5-3.0 \mathrm{kGy}$. The standard does not deliver any information on the identity of the EPR signals involved. Currently the European standard for the detection of irradiated food containing crystalline sugars is widely used in many food control laboratories around Europe and the world.

Several works have been published to identify radicals responsible for the multiline EPR signal in dried fruits and sugars by applying the sophisticated EPR and ENDOR analyses $[10,11]$. The authors of present study identified the EPR spectra derived from different sugar components in irradiated fruits $[12,13]$.

In various fresh fruits, sugar content is different [14]. Consequently, the same concerns dried fruits which contain different concentration of sugar which appears typically in a crystalline form. The presence of sugar crystals in dried fruits is essential since free radicals trapped only in crystalline phase are stable in contrast to those produced by radiation in liquid or glassy states. The latter recombine within a few seconds or minutes after irradiation. The higher the content of crystalline sugar in dried fruit the more intense EPR signal is observed.

The content of sugars in fresh fruits studied is given in Table 1.

All fruits imported from Asia and South America to Europe and USA undergo routine quarantine to avoid the migration of dangerous insects to these continents. In order to accelerate the quarantine of fruits, fumigation or recently, more and more widely, the treatment with low dose of ionizing radiation of the order of 200-300 Gy is applied. The latter method is more safe (no harmful deposits) and fully effective. The problem is whether quarantine doses can be detected by standardized analytical method.

\section{Materials and methods}

Three kinds of fresh fruits - pineapple, banana and papaya, and two kinds of commercially available dried fruits - fig and California plum were purchased in the market. The samples of fructose and glucose of analytical purity were delivered from Aldrich. All

Table 1. The content of sugars in fresh fruit in grams for $100 \mathrm{~g}$ of fresh fruit

\begin{tabular}{lccc}
\hline \multicolumn{1}{c}{ Fruit } & Carbohydrates & Fructose & Glucose \\
\hline Pineapple & 13.1 & 2.1 & 2.9 \\
California plum* & 11.4 & 3.1 & 5.1 \\
Banana & 22.8 & 2.7 & 4.2 \\
Papaya & 13.5 & 1.4 & 2.7 \\
Fig* & 63.9 & 24.4 & 26.9 \\
\hline
\end{tabular}

\footnotetext{
* Undergone to drying process
}

fruit samples were dried for $48 \mathrm{~h}$ at $40^{\circ} \mathrm{C}$ and subsequently kept in the exsiccator under the vacuum to avoid contact with moisture and dust. The samples were irradiated at room temperature with the doses of $0.5,1.0$, and $3.0 \mathrm{kGy}$ in the ${ }^{60} \mathrm{Co}$ source Gamma Chamber 5000. The EPR spectra were recorded at room temperature with Bruker ESP $300 \mathrm{X}$ band spectrometer one day after irradiation and then after 30, 90, 180, and 360 days. Spectrometer settings were adjusted as recommended in EN 13708 European standard. The EPR signal intensity was obtained by the integration of recorded EPR signal areas within $6 \mathrm{mT}$ of magnetic field range.

\section{Results and discussion}

The irradiated samples of pineapple, banana, papaya and fig showed the broad, multiline EPR signals specific for fruits sugars [9]. The exception was the sample of dried California plum for which no EPR signal was detected after irradiation. The widths of these signals, changing from $5.5 \pm 0.2 \mathrm{mT}$ for banana to $6.2 \pm 0.2 \mathrm{mT}$ for papaya, are consistent with the data published earlier for other fruits sugars $[4,13,14]$.

The advantage of the EPR method for detection of dried fruits irradiation is an easy distinction of the signals from irradiated and nonirradiated samples. Some of the unirradiated fruits show weak, narrow singlets in contrast to broad, intensive and multiline signals of irradiated fruits. The EPR signals of unirradiated and irradiated dried pineapple are shown in Fig. 1. Unirradiated pineapple does not show any EPR signal. After irradiation with the doses of 0.5 and $3.0 \mathrm{kGy}$, the broad doublet AA-BB with hyperfine splitting of about $1.1 \mathrm{mT}$ is detected. Both lines of doublet are superimposed with unresolved sharp lines. The intensity of EPR
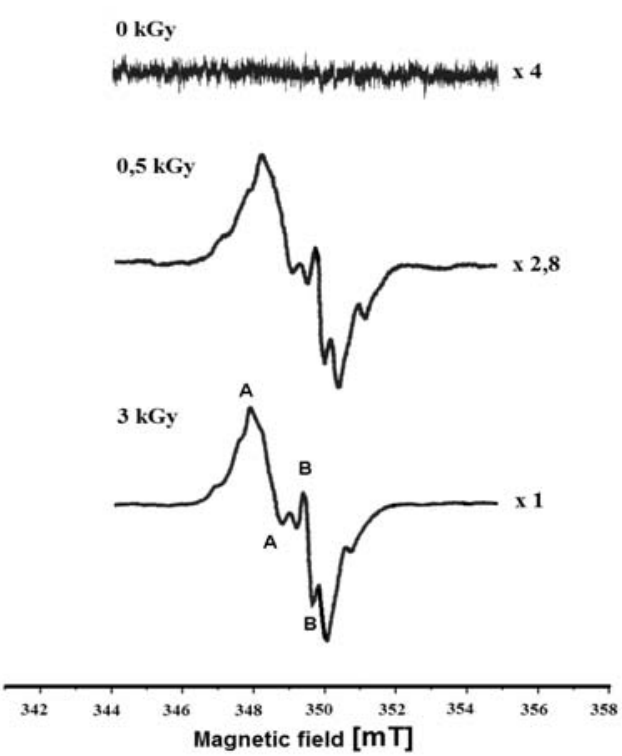

Fig. 1. EPR signals of dried pineapple unirradiated and irradiated with doses of 0.5 and $3 \mathrm{kGy}$. The numbers on the right refer to attenuation. The signals were recorded one month after irradiation. 

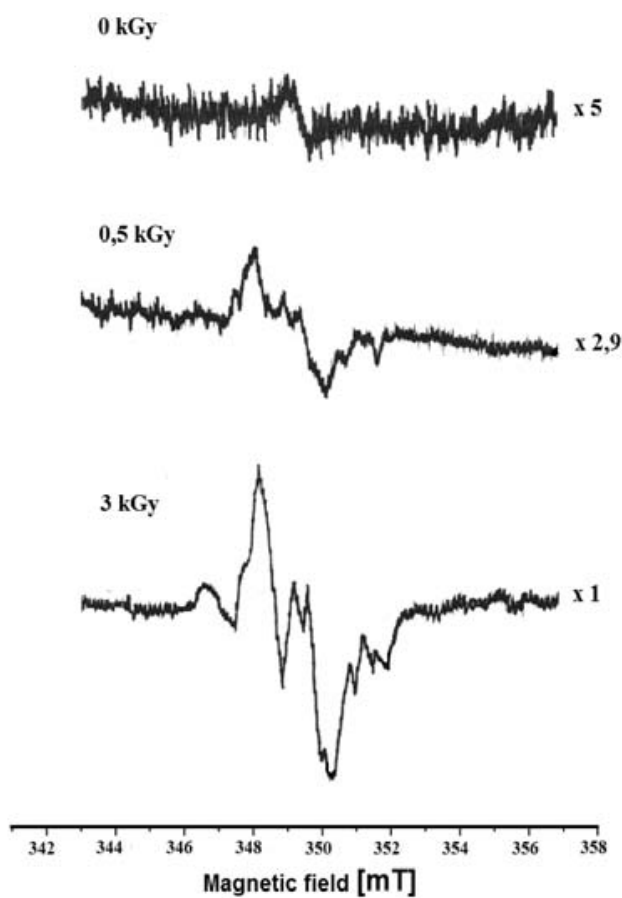

Fig. 2. EPR signals of dried fig unirradiated and irradiated with doses of 0.5 and $3 \mathrm{kGy}$. The numbers on the right denote attenuation. The signals were recorded one month after irradiation.

signal of sample irradiated with a dose of $0.5 \mathrm{kGy}$ is nearly 3 times lower than that of a sample irradiated with the dose of $3.0 \mathrm{kGy}$. However, dried pineapple irradiated with $0.5 \mathrm{kGy}$ can be easily identified by EPR as an irradiated sample.

Figure 2 shows the EPR spectra of unirradiated and irradiated samples of dried fig. In this case a weak native singlet with $g=2.004$ appears for an unirradiated sample. After irradiation with a dose of $0.5 \mathrm{kGy}$, multiline signal is recorded. The amplitude of this signal is only twice as higher than that of the native singlet. However, the integrated area of this signal exceeds the area of the native signal about 20 times making it possible for the reliable distinction between both signals. It means that detection of low dose radiation treatment $(0.5 \mathrm{kGy}$ and lower, i.e. $200-300 \mathrm{~Gy}$ ) of fig is fully possible. It is clear, therefore, that EPR method is suitable for the detection of dried fruits exposed to quarantine treatment with the use of ionizing radiation. The EPR signal recorded after irradiation with a dose of $3 \mathrm{kGy}$ is more intense and better resolved. To some extent, its shape resembles the EPR signal of irradiated pineapple.

The EPR spectra of irradiated dried banana and papaya (not shown in this paper) are also dominated by a broad doublet with hyperfine splitting of $1.3 \mathrm{mT}$ for banana and $1.1 \mathrm{mT}$ for papaya resembling the EPR spectra of pineapple and fig.

It seems reasonable to assume that observed spectral similarities are due to the sugar composition in studied dried fruits. In pineapple, fig, banana and papaya, the dominating sugars are fructose and glucose appearing in proportion 1:1.

The EPR spectra of commercial fructose and glucose irradiated with a dose of $3 \mathrm{kGy}$ are shown in Fig. 3.
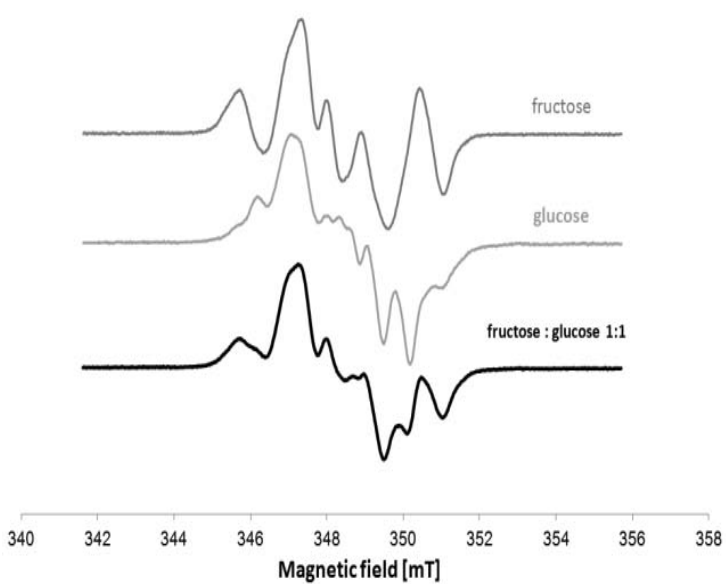

Fig. 3. The experimental EPR spectra of irradiated fructose and glucose. Spectrum on the top is superposition of both spectra with intensity ratio $1: 1$.

The shape of both spectra is complex indicating the contribution of EPR signals of different radicals. The superposition of EPR spectra of fructose and glucose with intensity ratio 1:1 produce the signal which resembles to some extent the experimental spectra of irradiated dried pineapple and fig samples. Thus, it can be concluded that major paramagnetic products stabilized in dried pineapple and fig are free radicals originated from fructose and glucose. Similar proportion between glucose and fructose contents and between glucose and fructose radical yields in irradiated fruits may indicate that stable radicals are formed in primary stages of radiolysis. The alternative supposition that those radicals are side products of secondary radical transformation [15] seems less probable, because the composition and yield of radiolytic molecular products are entirely different for glucose and fructose.

It has to be noted that the EPR signal of irradiated fig shows distinctly lower intensity as compared with the signal of irradiated pineapple, although sugar content in the latter is higher. Fresh pineapple contains only $2.1 \mathrm{~g}$ of fructose and $2.9 \mathrm{~g}$ of glucose in $100 \mathrm{~g}$ while $100 \mathrm{~g}$ of commercially dried fig contains as much as $24.4 \mathrm{~g}$ of fructose and $26.9 \mathrm{~g}$ of glucose, respectively. The unexpectedly low intensity of EPR signal of irradiated fig in comparison with that of pineapple is probably caused by a low content of crystalline fraction in the total sugar pool. The drying process of fresh fruits proceeds in a different way in specific species. Depending on initial water content, thickness of fruit and its porosity, as well as drying temperature, pressure, and duration of the process, the content of crystalline sugar fraction in dried fruit can vary in a significant way. The condition of fruit and all factors mentioned above influence the crystallization efficiency and the dimensions of sugar crystallites formed. Since radiation induced stable radicals are stabilized in crystalline sugars only, the total sugar content in fruits does not determine the EPR signal intensity of irradiated fruits.

The samples of dried California plums do not show any EPR signal after irradiation. It is supposed that in this fruit even after intense drying sugars re- 


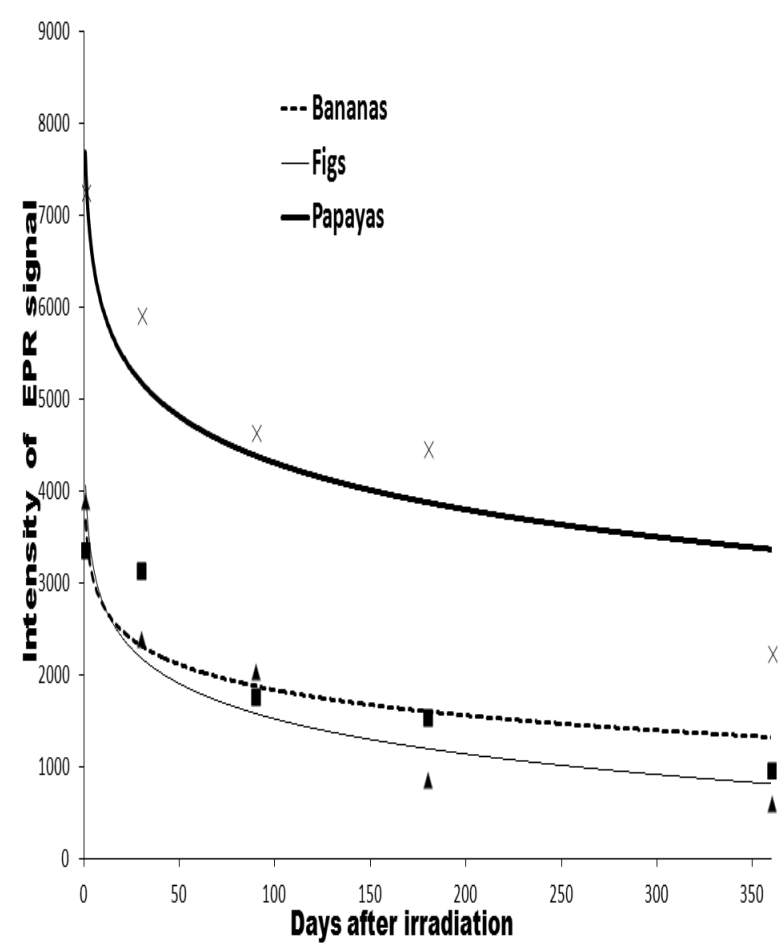

Fig. 4. Decay of the EPR signals of dried fruits irradiated with the dose of $1 \mathrm{kGy}$. Banana $\boldsymbol{\bullet}$, fig $\boldsymbol{\Lambda}$, and papaya $\mathbf{x}$. main dispersed in the pulp in a form of syrup which never crystallizes.

The stability of EPR signals derived from radicals produced by radiation in dried fruits is limited. For that reason, to evaluate the detection ability of the EPR method after prolonged storage of irradiated fruits, the time dependent kinetic study has been carried out.

Figure 4 shows the decay curves of dried papaya, banana, and fig irradiated with the dose of $1 \mathrm{kGy}$. The intensity of EPR signals decreases sharply during the first month after irradiation. Then the decay becomes gradually slower, however after one year of storage, the EPR signal intensity remains still relatively high. For fig and banana, it is about $40 \%$ of initial intensity as measured one day after irradiation. For pineapple and papaya, the decay is larger reaching about $80 \%$.

Thus, it can be concluded that the intensities of EPR signals of irradiated fruits after one year of storage are sufficient to prove the fact of radiation treatment. It means that the EPR method for the detection of irradiated fruits is a useful and reliable technique for the control of dried fruits exposed to radiation.

The dependence of the EPR signals intensity vs. radiation dose as measured one year after irradiation is shown in Fig. 5. For all fruits investigated, the signal intensity increases with radiation dose for the range $0-3 \mathrm{kGy}$. The best fit has been obtained for linear function determined by the highest $R$-square coefficients which for bananas are $R^{2}=0.9299$, for papaya $R^{2}=0.8749$, while for fig $R^{2}=0.9132$. The linear relationship of EPR signal intensity vs. dose has been observed earlier for irradiated dried grapefruits [16] and dried candied fruits, the components of diet supplements [17].

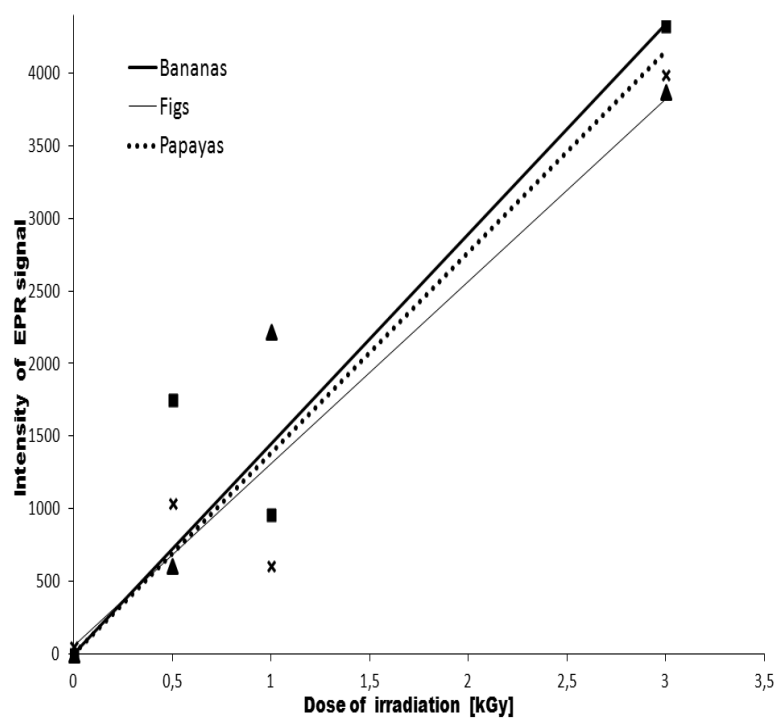

Fig. 5. Dose dependence of EPR signal recorded one year after irradiation of dried fruits. Banana $\mathbf{\square}$, fig $\mathbf{x}$, and papaya $\boldsymbol{\Lambda}$

\section{Conclusions}

The irradiation of dried banana, pineapple, papaya, and fig generates free radical trapped in sugar crystallites present in dried fruits. The complex EPR spectra of these radicals can be detected even one year after irradiation. The intensity of EPR spectra of irradiated dried fruits exceeds markedly the intensity of native signals in unirradiated fruits. It was proven that glucose and fructose radicals are the major paramagnetic products in irradiated banana, pineapple, papaya and fig samples. The recorded EPR spectra can be reconstructed in the best way by superposition of the spectra of irradiated glucose and fructose with the intensity ratio $1: 1$. At room temperature, the EPR signals decrease quite rapidly during the first month after irradiation. The decay during the following months is distinctly slower. It was found that the EPR signals recorded one year after irradiation are sufficiently intensive for effective control of dried fruits irradiated with the doses below $0.5 \mathrm{kGy}$. Thus, the EPR technique can be considered as a very reliable method for the control of dried fruits after prolonged storage (1-3 years) even when irradiated with low doses including quarantine treatment (200-300 Gy). Dried California plum did not show EPR signal presumably due to the lack of crystalline sugar and for that reason cannot be controlled by the EPR method. The results of present study are of practical use and throw more light on the nature of radicals stabilized in irradiated dried fruits.

\section{References}

1. Commoner, B., Townsed, J., \& Pake, G. E. (1954). Agricultural research under review. Nature, 174, 663-665.

2. Dood, N. J. F., Swallow, A. J., \& Ley, F. J. (1985). Use of ESR to identify irradiated food. Radiat. Phys. Chem., 26, 451-453.

3. Raffi, J., Agnel, J. -P., Buscarlet, L. A., \& Martin, C. C. (1988). Electron spin resonance identification of 
irradiated strawberries. J. Chem. Soc., Faraday Trans., 1, 84, 3359-3362.

4. Raffi, J., \& Agnel, J. -P. (1989). Electron spin resonance identification of irradiated fruits. Radiat. Phys. Chem., 34(6), 891-894.

5. Raffi, J., Stachowicz, W., Migdał, W., Barabassy, S., Kalman, B., Yordanov, N., Andrade, E., Prost, M., \& Callens, F. (1998). Establishment of an eastern network of laboratories for identification of irradiated foodstuffs. Final Report of Copernicus Concerned Action. CCE. (CIPA-CT94-0134).

6. Raffi, J., Stevenson, M. H., Kent, M., Thiery, J. M., \& Belliardo, J. -J. (1992). European intercomparison on electron spin resonance identification of irradiated foodstuffs. Int. J. Food Sci. Technol., 27, 111-124.

7. Joint FAO/IAEA/WHO Study Group. (1999). High-dose irradiation: Wholesomeness of food irradiated with doses above 10 kGy. Geneva: World Health Organization.

8. Linke, B., Ammon, J., Ballin, U., Brockmann, R., Brunner, J., Delincee, H., Eisen, S., Eming, D., Eschelbach, H., Estendorfer-Rinner, S., Fienitz, B., Frohmut, G., Helle, N., Holstein, K., Jonas, K., Krolls, W., Kuhn, T., Kruspe, W., Marchioni, E., Meier, W., Pford, J., Schleich, C., Stewart, E., Trapp, C., Vreden, N., Wiezorek, C., Bogl, K. W., \& Schreiber, G. A. (1996). Elektronenspinresonanzspektro-skopische Untersuchungen zur Identifizierungbestrahlter getrockneter und trischer Fruchte: Durchführung eines Ringversuches an getrockneten Feigen und Mangos sowie an frischen Erdbeeren. Berlin: Bundesinstitut für gesundheitlichen Verbrau-cherschutz und Veterinärmedizin. (BgW - Hette 03/1996).

9. EN 13708:2003: Foodstuffs - Detection of irradiated food containing crystalline sugar by ESR spectros- copy. Brussels: European Committee for Standardisation $(\mathrm{CEN})$

10. Vanhaelewyn, G., Jansen, B., Callens, F. J., \& Sagstuen, E. (2004). ENDOR - Assisted study of the stable EPR spectrum of X-irradiated $\alpha$-L-Sorbose single crystals: MLCFA and simulation decomposition analyses. Radiat. Res., 162, 96-104.

11. Vanhaelewyn, G., Sadło, J., Callens, F., Mondelaers, W., Frenne, D., \& Matthys, P. (2000). A decomposition study of the EPR spectrum of irradiated sucrose. Appl. Radiat. Isot., 52, 1221-1227.

12. Guzik, G. P., Stachowicz, W., \& Michalik, J. (2008). Study on stable radicals produced by ionising radiation in dried fruits and related sugars by electron paramagnetic resonance spectrometry and photostimulated luminescence method I. D-fructose. Nukleonika, 53 (Suppl. 2), S89-S94.

13. Guzik, G. P., \& Stachowicz, W. (2013). Study on radicals giving rise to multicomponent EMR spectra in dried fruits exposed to ionizing radiation II. D(+) Glucose. Nukleonika, 58(3), 425-428.

14. The physicochemical guide. The group work in Polish. (1974). Warsaw: Wydawnictwa Naukowo-Techniczne WNT.

15. Von Sonntag, C., \& Schuchmann, H. -P. (2001). Studies in physical and theoretical chemistry: Carbohydrates. In Radiation chemistry: Present status and future trends (pp. 481-511). Elsevier Science.

16. Tabner, B. J., \& Tabner, V. A. (1991). An electron spin resonance study of gamma-irradiated grapes. Radiat. Phys. Chem., 38(6), 523-531.

17. Helle, N., Linke, B., Schroeiber, G. A., \& Bogl, K. W. (1992). Nachweis der gamma Bestrahlung von Trockenfrüchten. Bundesgesundheitsblatt, 35, 179-184. 